\title{
Broadband Delay System Demonstration for VLBI2010
}

\author{
Arthur Niell ${ }^{1}$ \\ MIT Haystack Observatory \\ Off Rte 40, Westford, MA, USA 01886 \\ E-mail: aniellehaystack.mit.edu
}

\begin{abstract}
VLBI2010 is the next generation geodetic VLBI system being developed under the auspices of the IVS. This system is envisioned to make use of comparatively small but cost-effective $12-\mathrm{m}$ class antennas together with very broadband feeds $(2-14 \mathrm{GHz})$ and multiple IF channels to reliably resolve RF phase. In order to demonstrate that the "broadband delay" concept is feasible, all of the components of the broadband delay system have been implemented on the Westford $18 \mathrm{~m}$ and MV3 $5 \mathrm{~m}$ antennas. The combined sensitivity of these two antennas is somewhat less than that of two $12 \mathrm{~m}$ antennas but should be sufficient to demonstrate that the concept can be achieved. As configured the interferometer is capable of observing four 512 MHz bands within the frequency range $3.3 \mathrm{GHz}$ to $11.5 \mathrm{GHz}$ in both linear polarizations with an aggregate data rate of 8 gigabits per second. Observations to date show that the instrumentation functions as expected, but the sensitivities of the antennas are lower than anticipated. Efforts are underway to optimize the installation and operation of the cooled feed and receivers on both antennas.
\end{abstract}

The 9th European VLBI Network Symposium on

The role of VLBI in the Golden Age for Radio Astronomy and EVN Users Meeting

Bologna, Italy

September 23-26, 2008

\footnotetext{
${ }^{1}$ Speaker
} 


\section{Introduction}

Very Long Baseline Interferometry (VLBI), along with Satellite Laser Ranging, GPS, and DORIS, measure the shape of the Earth and its orientation in space. VLBI provides the Celestial Reference Frame as defined by the extragalactic radio objects that are the source of the observed radio emission, the orientation of the spin axis in inertial space, and time, and contributes significantly to determining the scale of the Earth's size. The current accuracy of the Terrestrial Reference Frame is about one centimeter, but the desired accuracy is one millimeter.

VLBI accuracy is limited primarily by the error in determining the effect of the neutral atmosphere. This is due at least in part to the low speed of the existing antennas, which does not allow rapid sampling of the atmosphere. Another limitation is the lack of a sufficient number of globally distributed antennas. From recent studies the minimum number is around sixteen. The present network is concentrated in the northern hemisphere.

The geodetic VLBI observing system is also suffering from age. Many of the antennas are requiring increasing amounts of maintenance, and several have already had to be removed from service.

Another limitation in the present system is the loss of signal due to radio frequency intereference (RFI) in the bands that are observed. The current hardware does not allow changing frequency sufficiently to avoid this problem, which will only increase with time.

A study by a working group within the International VLBI Service (IVS) recommended that construction of a new array of antennas be initiated that would address all of these issues. The characteristics of the antennas would be high slew rates, flexible choice of frequency reception in the range from the current S-band (approximately $2.2 \mathrm{GHz}$ ) up to approximately 15 $\mathrm{GHz}$, and the ability to record data in four or more bands separately. Fast slewing is needed to sample the atmosphere in different directions very often in order to reduce the error due to the rapid variations in atmospheric delay, both temporally and spatially. By recording at very high rates compared to the current observations, smaller and thus less expensive antennas could utilized. The ability to record four frequency bands should enable the use of phase delay as the observable, which is intrinsically more precise than the current group delay by almost an order of magnitude for the frequency range proposed.

In order to evaluate the recommended solution, funding has been provided by $\mathrm{NASA}^{1}$ for the construction and testing of a proof-of-concept demonstration system to be installed on two existing antennas of the geodetic network. Several recent developments have encouraged the initiation of such a demonstration at this time: a) recent improvements in high data rate recording capability, b) the ready availability of wide-band $(\sim 10 \mathrm{GHz})$ low-noise amplifiers, and c) access to apprpriate digital back ends. Another development that will make the proposed system feasible operationally is the increased speed and capacity of software correlators, which will allow the higher data rates and larger networks to be correlated economically and flexibly.

\footnotetext{
${ }^{1}$ The proposal was funded by NASA's Earth Surface and Interior Focus Area through the efforts of John Labrecque, Chopo Ma, and Herb Frey
} 
The characteristics of the demonstration system, some potential new limitations, and the initial testing are described in this article.

\section{Broadband delay concept}

Up to the present and continuing, the ionosphere-corrected delay observable for geodetic VLBI has been estimated from the group delays measured at S-band (2.2-2.4 GHz) and X-band $(8.1 \mathrm{GHz}-8.9 \mathrm{GHz})$. The group delay at each band is estimated from data taken in sub-bands with bandwidths of $2 \mathrm{MHz}$ to $16 \mathrm{MHz}$, which is conventionally called the multi-band delay. The use of more than two frequencies to determine the phase delay observable by connecting phase across the total spanned bandwidth is being called broadband delay (BBD) to distinguish it from multi-band delay. With four frequencies spanning the range from approximately 2.2 $\mathrm{GHz}$ to $11 \mathrm{GHz}$ it is possible to reliably estimate the phase delay in the presence of the ionosphere at a total signal-to-noise ratio (SNR) of 20 or less (Petrachenko et al IVS memo). While a wider frequency range, especially extending to higher frequencies, could be utilized, the range is limited for the demonstration by hardware design, especially of the local oscillator (UpDown Converter).

A possible limitation to the effectiveness of using the BBD is the variation of phase with frequency and with baseline length and orientation due to the structures of the radio sources. Simulations suggest that this may not be a problem for sources with simple enough structure, but a more likely solution that will increase the number of usable sources is to determine the structures from the observations themselves. With high precision delays determined from very short scans, there will a very large number of observations per day on each source from which the structure can be estimated.

\section{BBD demonstrator system}

The principal components of the broadband delay system are the broadband feed, RF filters, phase calibrator, broadband low noise amplifiers, optical fiber connection from the antenna to the control room, splitters, flexible local oscillators, digital back end, and recorders. Most of these components are indicated in Figure 1 (next page).

For this demonstration both linear polarizations are recorded for each of four $512 \mathrm{MHz}$ bands taken somewhere in the frequency range from approximately $1 \mathrm{GHz}$ to $13 \mathrm{GHz}$. Each $512 \mathrm{MHz}$ band is filtered by the digital back end (DBE) to obtain sixteen 32-MHz channels, and the odd channels are output giving a data rate of 1 gigabit/second (Gbps) in each bandpolarization, yielding a total data rate of $8 \mathrm{Gbps}$. The choice to record only half of the data in each band-polarization was made for the practical reason of keeping the number of frequency converters, DBEs, and recorders to four each.Recording the full bands to give 16 Gbps would have doubled the number of components.

Aside from the antenna the components of the broadband system are the broadband feed, RF filters, phase calibrator, low noise amplifiers, optical fiber connection from the antenna to the control room, splitters, flexible local oscillators, digital back end, and recorders. 


\subsection{Front end}

In order to reduce the system temperature the feed, filters, directional couplers for phase cal injection, and LNAs are located inside the Dewar. The physical temperatures of the feed and LNAs are approximately 20K. The feed is an ETS-Lindgren model 3164-05 dual linear polarization feed with a nominal frequency range of 2-18 GHz. The broadband MMIC LNAs were obtained through Caltech. For the design of the Dewar and the choice of feed and LNAs we gratefully acknowledge the work of Sandy Weinreb of Caltech and the continued support by both Sandy and Hamdi Mani of Caltech.

Since the tunnel diodes on which the earlier Mark 4 phase calibrator was based are no longer available, it has been necessary to develop a new method for generating the extremely short pulses needed to produce harmonics up to $12 \mathrm{GHz}$ for the broadband system The phase calibrator to be used for the BBD proof-of-concept is a new design by Alan Rogers that uses an ultrafast digital comparator to generate the short pulses and is described in Haystack Observatory Mark5 Memo 75 (http://www.haystack.mit.edu/tech/vlbi/mark5/memo.html).

The goal of VLBI2010 is to use frequencies down to $2.2 \mathrm{GHz}$ so that compatible observations can be made with the current geodetic antennas at S-band and X-band. However, RFI from sources below $1 \mathrm{GHz}$ were found to saturate the front end, so high-pass filters were installed before the phase cal injection and the LNAs. For the current observing the lower frequency bound is approximately $3 \mathrm{GHz}$. In the future custom filters will be obtained to provide the necessary sharp cutoff just below $2.2 \mathrm{GHz}$.

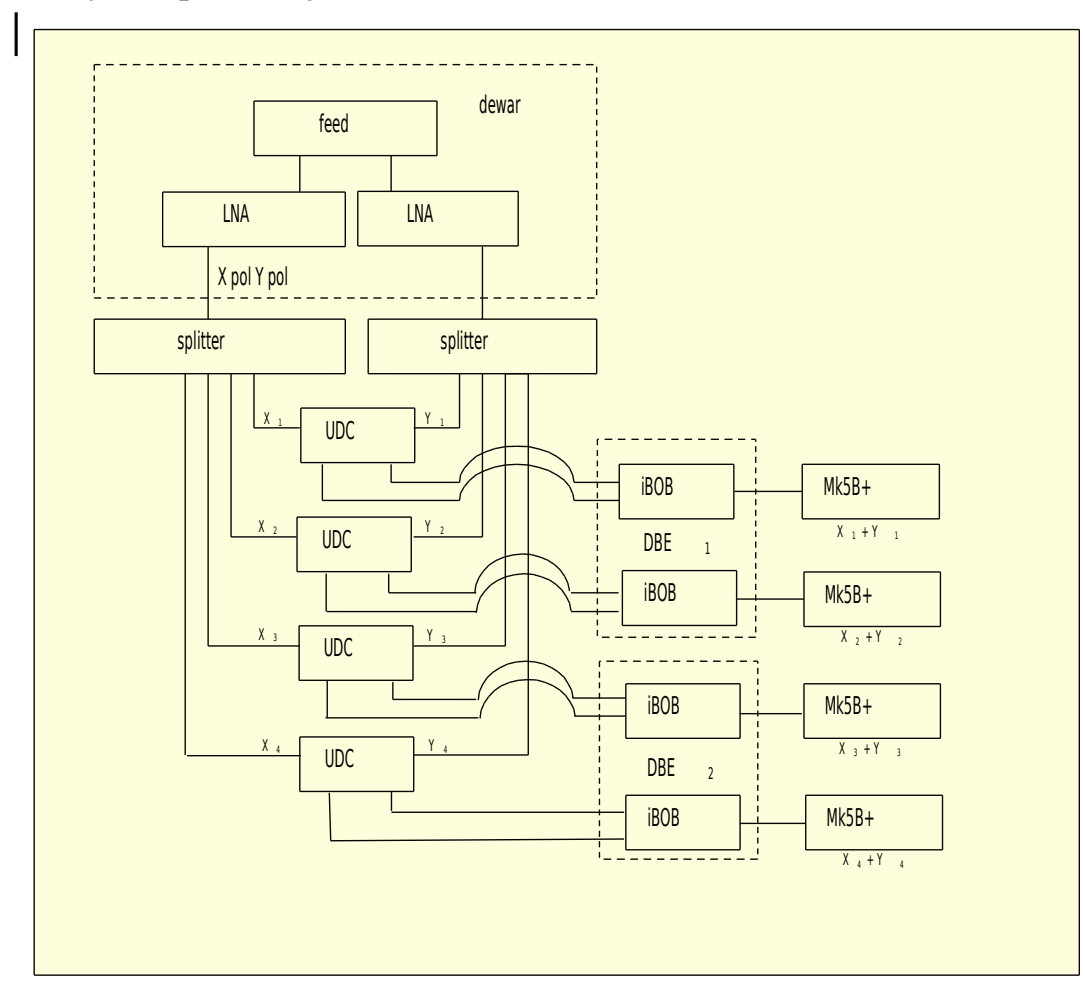

Figure 1. Diagram of broadband system components. LNA: low noise amplifier; UDC: flexible local oscillator; DBE: digital back end; iBOB (developed by 
CASPER group, UC Berkeley): main board of the DBE; Mk5B+: disk-based 2 Gbps recorder. All elements outside the Dewar are in the control room.

\subsection{Back end}

The broadband RF signals for each polarization are brought down from the antenna to the control room on optical fiber to minimize sensitivity to temperature change and mechanical stress on the downlink. The fiber receivers, additional amplifiers, and four-way splitters are in one chassis. The outputs from the splitters are routed to the UDCs, DBEs, and recorders, all located in one $1.8 \mathrm{~m}$ rack.

\subsubsection{UpDown Converters}

A major requirement of the VLBI2010 system is that the frequencies of the bands be selectable. This is because radio frequency interference (RFI) is increasing, and it is desirable to be able to change frequencies flexibly. This has been implemented by Alan Rogers in an UpDown Converter which uses a first variable frequency local oscillator (LO) to raise the selected band to approximately $22.5 \mathrm{GHz}$ then a fixed frequency LO to down-convert to a 2 $\mathrm{GHz}$ passband at video. Two input channels are converted by the same LOs, making the UDC ideal for the two linear polarization channels. For each input channel it is possible to select two output Nyquist zones, each of which can be amplified by $30-60 \mathrm{~dB}$. The UDC and its specifications are described in Mk5 Memos 56, 59, 60, and 70.

\subsubsection{Digital back end and recorder}

The DBEs are based on the iBOB board developed by the CASPER group of UC Berkeley with fpga code developed at Haystack Observatory. Two IFs (two polarizations from the same band) are input to the analog-to-digital converter of each DBE/iBOB. After the polyphase filter half of the sixteen $32 \mathrm{MHz}$ wide channels from each polarization are routed onto one VSI-H output which is then fed to one Mark5B + recorder. For these demonstrations the disk modules of the Mark5B+ are populated with either four terabytes (TB) or six terabytes of disk capacity. The disk consumption is approximately $1 \mathrm{~TB}$ per hour for each Mark5B + when recording continuously.

\subsection{Antennas}

The antennas used for the proof-of-concept demonstration are the 18 meter prime focus antenna located near Westford, Massachusetts, USA, and the 5 meter MV3 antenna located in Greenbelt, Maryland, USA, at the Goddard Space Flight Center. At Westford there was no option but to mount the Dewar at prime focus, which is the location of the standard geodetic S/ $\mathrm{X}$ feed. At MV3 the choice was made to mount the Dewar at Cassegrain, also as used for Xband in the $\mathrm{S} / \mathrm{X}$ system. The Lindgren feed in the Dewar is not well matched to either antenna because of its small beamwidth, resulting in lower efficiencies than obtained for S/X operation. Because of the low efficiencies, determination of the proper focus settings and pointing corrections is proving difficult. 


\section{Status of demonstration}

Except for the new digital phase calibration unit, all components have been installed and tested. Mk4 phase cal units are currently being used with a modification to generate $5 \mathrm{MHz}$ rail spacing rather than the $1 \mathrm{MHz}$ spacing generated for standard $\mathrm{S} / \mathrm{X}$ observations.

Fringes have been reliably detected in both polarizations of all four channels. Most sessions have been conducted at X-band with considerable redundancy of settings in order to verify that all components are working properly.

In a recent session the full frequency range from $3.3 \mathrm{GHz}$ to $11.5 \mathrm{GHz}$ was covered in four scans by setting the four $512 \mathrm{MHz}$ bands to be contiguous in each scan. In previous experiments only frequencies in the $8-9 \mathrm{GHz}$ range were recorded. In these first observations in the 3-8 GHz range strong detections were obtained. However, this was at the expense of the Xband response which appears to have suffered when the focus was adjusted to optimize the 3.7 $\mathrm{GHz}$ sensitivity. The data are only partially correlated and analyzed, so these results are preliminary.

\section{Plans}

Although the broadband system instrumentation functions as anticipated, the installation of the Dewars on the antennas must be understood better in order to improve the sensitivity through focus adjustments and pointing corrections. In particular focus settings must be determined that optimize the sensitivity across the $3-11 \mathrm{GHz}$ range.

An important challenge that must be addressed is how best to obtain the delay observable from linear polarizations; all previous geodetic observing has used circular polarization. The broadband concept anticipates that with the four bands it will be possible to resolve the phase ambiguities in the presence of the ionosphere to obtain a true phase delay. Simulations indicate that, in the absence of phase variations due to source structure, this can be accomplished for a total SNR of less than 20. Observations with this demonstrator will be used to investigate the effects of source structure on the phase across a continuous approximately $8 \mathrm{GHz}$ band.

The eventual goal is to do a series of geodetic sessions to evaluate the success of the broadband delay measurement system, thus validating the VLBI2010 concept of using small fast antennas, high data rates, and multiple frequency bands to significantly improve the accuracy of geodetic VLBI.

\section{Acknowledgements}

This is a team project with important contributions by all participants: Bruce Whittier ${ }^{1}$, Mike Titus ${ }^{1}$, Jason SooHoo ${ }^{1}$, Dan Smythe ${ }^{1}$, Alan Rogers ${ }^{1}$, Jay Redmond ${ }^{2}$, Mike Poirier ${ }^{1}$, Chuck Kodak, Alan Hinton ${ }^{1}$, Ed Himwich ${ }^{3}$, Skip Gordon ${ }^{2}$, Mark Evangelista ${ }^{2}$, Irv Diegel ${ }^{2}$, Brian Corey $^{1}$, Tom Clark ${ }^{3}$, and Chris Beaudoin ${ }^{1}$. We also want to thank Dan MacMillan, Peter Bolis, Don Sousa, and Dave Fields for their help, and Shep Doeleman of Haystack for his significant contributions to the successful implementation of the DBE.

${ }^{1}$ MIT Haystack Observatory ${ }^{2}$ Honeywell Technical Services, Inc. ${ }^{3} \mathrm{NVI} / \mathrm{GSFC}$ 